\title{
educação

\section{Educar para el cambio y la sostenibilidad: Evaluación de una propuesta de aprendizaje experiencial para formar al profesorado en formación inicial}

\author{
Mercedes Varela-Losada ${ }^{1} \&$ Azucena Arias- \\ CORREA II \\ Universidade de Vigo, España \\ PEDRO Vega-Marcote ${ }^{\mathrm{III}}$ \\ Universidade da Coruña, España
}

\begin{abstract}
Uno de los principales retos que debe afrontar la educación es la búsqueda de la Sostenibilidad. Y, en este proceso, el profesorado es un pilar fundamental. Por ello, se presenta una propuesta educativa de formación inicial de profesorado basada en la experimentación y análisis de un ciclo de aprendizaje en torno a la problemática socioambiental del cambio climático. Para su evaluación se utilizó una metodología de carácter mixto. Los resultados obtenidos sugieren que este tipo de prácticas pueden contribuir a mejorar el compromiso del futuro profesorado con una Educación Ambiental orientada hacia la Sostenibilidad y crítica con el modelo actual de desarrollo, permitiendo la construcción de un marco educativo basado en metodologías globalizadas y participativas, así como a favorecer la adquisición de competencias docentes.
\end{abstract}

Palabras clave: Educación Ambiental y Sostenibilidad; Formación del profesorado; Aprendizaje experiencial; Ciclo de aprendizaje

\section{INTRODUCCIÓN}

En el inicio del siglo XXI nos encontramos en medio de una gran crisis ambiental y humana, que nos lleva hacia un Cambio Ambiental Global que está alterando la estructura y el funcionamiento del complejo Sistema Tierra (Steffen \& Stafford, 2013), con un gran coste para los ecosistemas, los recursos del planeta y el clima (Worldwatch Institute, 2015). Por tanto, este es uno de los principales retos que debe afrontar la educación del siglo XXI, tal como reflejan los "Objetivos de Desarrollo Sostenible del Milenio" (Naciones Unidas,
2015).

No obstante, la promoción de estilos de vida sostenibles es un objetivo enormemente complejo (Stern, 2000), como se refleja en la variabilidad de corrientes y modelos pedagógicos que existen en la Educación Ambiental. En los últimos años, diversas autoridades en este campo proponen enfoques hacia la acción, fortalecida por el pensamiento crítico y la participación democrática (Mogensen \& Mayer, 2005; Piasentin \& Roberts, 2018) y una búsqueda del Desarrollo Sostenible (Kopnina, 2012). Es necesaria, pues, una Educación Ambiental orientada hacia 
la Sostenibilidad (de aquí en adelante EAS) con una visión crítica y transformadora que contribuya a alcanzar una forma de progreso más justa y sostenible (Lotz-Sisitka, Wals, Kronlid, \& McGarry, 2015).

Por tanto, las escuelas deben sufrir un proceso de transformación (Tilbury, 2011), donde el profesorado es un pilar fundamental, por su responsabilidad directa en el proceso y por su contacto diario con el alumnado. En este proceso están documentadas barreras y dificultades a las que el profesorado debe hacer frente para implementar iniciativas educativas sostenibles, que se podrían sintetizar como: barreras institucionales (Feinstein \& Kirchgasler, 2015; McKeown \& Hopkins, 2002), barreras formativas (García-Esteban \& Murga-Menoyo, 2015; Mascarell \& Vilches Peña, 2016) y barreras culturales (PérezRodríguez, Varela-Losada, Lorenzo-Rial, \& Vega-Marcote, 2017; Sund \& Wickman, 2011).

Las Universidades, como formadoras de profesorado, deberían proporcionar una formación consecuente con su rol de agentes de cambio del nuevo modelo de ciudadanía que demanda la sociedad, donde se debe introducir, entre otros, la dimensión de la Sostenibilidad (Alarcão, Tavares, Mealha, \& Souza, 2018). Sin embargo, la investigación muestra que la formación básica del profesorado presenta importantes debilidades en relación con la Educación Ambiental y la Sostenibilidad (García-Esteban \& MurgaMenoyo, 2015; Yavetz, Goldman, \& Pe'er, 2009). El profesorado, en formación inicial o en activo, sostiene ideas erróneas y conocimientos limitados sobre las cuestiones ambientales y la sostenibilidad, de sus componentes o de la forma en que estos componentes interactúan de una manera sistémica (p. ej. en Borg, Gericke, Höglund, \& Bergman, 2014; Desjean-Perrotta, Moseley, \& Cantu, 2008), y a veces no considera las cuestiones sociales y económicas como cuestiones fundamentales para la materia (Van Petegem, Blieck, \& Van Ongevalle, 2007). Destacar también que, a menudo, el personal docente no trata las cuestiones socioambientales en el aula, a pesar de que defienden su gran importancia (Agirreazkuenaga, 2019; Fortner \& Corney, 2002), esgrimiendo razones como su baja prioridad en los programas escolares (Marx \& Harris, 2006). Los estudios, asimismo, muestran el bajo nivel de conocimiento del profesorado y su inseguridad en relación con la enseñanza de la EAS (Eurydice, 2011), quejándose de falta de capacitación, tiempo y recursos para tratar temas tan relevantes y actuales como el cambio climático (Foss \& Ko, 2019). Así, el profesorado tiende a reproducir los modelos de enseñanza ya experimentados, lo que generalmente se traduce en una práctica tradicional transmisiva donde el estudiante es visto como un receptor pasivo (Eames et al. 2006; Garmendia Mujika, Barragués Fuentes, Zuza Elosegi, \& Guisasola Aranzábal, 2014). A todo esto se suma una preocupante discrepancia entre la filosofía de la EAS y la realidad de las práctica educativa, señalada por Stevenson (2007), que generalmente enfatiza la adquisición de conocimientos ambientales y la conciencia, cuando debería centrarse en la resolución de problemas y en objetivos orientados hacia la acción.

En la literatura existe una importante línea de investigación centrada en la innovación de la formación de profesorado en temas de sostenibilidad. La mayoría muestra propuestas centradas en el desarrollo de alfabetización ambiental y cambio de actitudes y modos de vida, como la diseñada por O 'Gorman y Davis (2013), que utilizan la huella ecológica como herramienta de cambio, o la de Fernández, Alférez, Vidal, Fernández, y Albareda (2016) con un proyecto multidisciplinar. Aunque también las hay centradas en el desarrollo 
de habilidades para la enseñanza de la EA, como la de Gayford (2001), que utiliza una metodología de investigación cooperativa, o la de Arreguín-Anderson y Kennedy (2013), que diseñaron una propuesta educativa basada en un programa de conservación de la vida salvaje. Sin embargo, revisando esta literatura parecen existir pocas experiencias que persigan el doble propósito de formar un profesorado comprometido con la conservación del medio y el bienestar de las personas, que a la vez posea las competencias docentes necesarias para promover entre su alumnado estas mismas cualidades, que es lo que se pretende con esta investigación.

\section{OBJETIVO DE LA INVESTIGaCión}

El objetivo general de la investigación fue evaluar una propuesta didáctica de Educación Ambiental orientada hacia la Sostenibilidad (de ahora en adelante EAS) para la formación del profesorado. Formulándose, para ello, dos cuestiones interrelacionadas: ¿Cuál es la contribución de una propuesta educativa basada en el aprendizaje experiencial para mejorar el compromiso del futuro profesorado con una EAS crítica con el modelo actual de desarrollo? ¿Y en qué medida se puede favorecer la construcción de un marco educativo propio para una EAS basada en el tratamiento de los problemas socioambientales desde una perspectiva crítica, la búsqueda de la acción participativa y responsable y la implicación de la comunidad?

\section{PROPUESTA DE FORMACIÓN EN EDUCACIÓN AMBIENTAL ORIENTADA A LA SOS TENIB ILIDAD}

La escuela debe preparar a las personas para comprender el mundo y repensarlo, de forma que se favorezcan modos de vida sostenibles. Por ello, la EAS debe basarse en un enfoque holístico y crítico hacia los modelos socioeconómicos preponderantes y las formas de vida actuales (Varela-Losada, Vega-Marcote, Pérez-Rodríguez, \& Álvarez-Lires, 2016).

Es, por tanto, un proceso complejo que conlleva la adquisición de conocimientos de tipo conceptual, pero también del desarrollo de valores, actitudes, aptitudes, y de maneras de actuar en interacción social, en colaboración y en diálogo (Tilbury, 2011). En este marco, es necesaria una adecuada formación del profesorado, que potencie la innovación y la reflexión, que busque formas de orientar el aprendizaje del alumnado alejadas de modelos transmisivos y basadas en el desarrollo de competencias (Cebrián \& Junyent, 2015). Es necesario un profesorado comprometido con la acción y la sostenibilidad, que sea consciente de la necesidad de cambio de nuestra forma de vida y de la importancia de fomentar lazos con la comunidad para impulsarlo.

Así, la propuesta de esta investigación fue diseñada específicamente para formación del profesorado y se fundamentó en las sugerencias de autoridades en el campo de la EAS, que deben ser la base del marco educativo del futuro profesorado. Por ello, se usó una metodología basada en la investigación de la sostenibilidad y en la resolución de problemas relevantes/ reales/cercanos, con planteamientos sustentados en la reflexión sobre la complejidad de las cuestiones ambientales (Mogensen \& Mayer, 2005; Varela-Losada et al., 2016; Wals, 2007). En la investigación se fomentó la alfabetización informacional y el desarrollo del pensamiento crítico, poniendo especial atención al rol de las personas en el sistema (Gifford, 2014; KyburzGraber, 2013; Varela-Losada et al., 2016; Wals, 2007). Asimismo se favoreció la reflexión sobre los modos de vida y los valores inconscientes asumidos y la toma de decisiones autónoma y responsable (Mogensen \& Mayer, 2005; Stern, 
Powell, \& Hill, 2014). Todo ello se llevó a cabo en un entorno social y participativo que buscaba motivar y favorecer una cultura democrática (Lave \& Wenger, 1991; Wals, 2007). Además se buscó integrar a la comunidad y la colaboración con el entorno (Vosniadou, 2001; Wals, 2007)

Para ello, se trataron, por una parte, cuestiones relacionadas con la sostenibilidad y, por otra, la forma de entender la EAS del futuro profesorado, de manera que estas dos dimensiones estuviesen interrelacionadas e integradas. El planteamiento se basó en el ciclo de aprendizaje de Karplus (Karplus, 1977), aunque adaptado a las concepciones antedichas (ver Figura 1).

\subsection{DESCRIPCIÓN DE LA PROPUESTA}

La propuesta, que aparece descrita detalladamente en Varela-Losada, Arias-Correa, y Vega-Marcote (2018), consistió en dos ciclos de aprendizaje que se desarrollaron de manera integrada, uno centrado en sostenibilidad y otro sobre las prácticas educativas en EAS.

Así, al inicio del proceso se favoreció la reflexión del alumnado sobre qué sabe y qué piensa sobre una EAS que facilite el cambio de los estilos de vida. Una primera puesta en común sirvió para propiciar una investigación sobre ello, donde se cuestionaron aspectos como los objetivos que

\section{Evaluación durante el Ciclo de aprendizaje sobre Educación Ambiental}

1. Exploración inicial

2. Introducción de nuevos conocimientos
- Escala de Actitudes hacia la Educación Ambiental (ASEE)

- Datos iniciales de las verbalizaciones del alumnado sobre cómo debe ser la Educación Ambiental

- Datos obtenidos del análisis del artículo científico sobre Educación Ambiental elaborado por el alumnado

\section{Ciclo de aprendizaje \\ sobre Sostenibilidad}

\section{Estructuración}

\section{Aplicación}

\section{Toma de datos final}

- Datos extraídos de las listas de ítems de análisis elaboradas por el alumnado (Cómo debe ser la Educación Ambiental)

- Datos obtenidos del análisis crítico sobre la adecuación de la propuesta experimentada (Ciclo de aprendizaje sobre Sostenibilidad) para un aula de Primaria elaborado por el alumnado

- Escala de Actitudes hacia la Educación Ambiental (ASEE)

- Entrevista semiestructuradas a un grupo representativo del profesorado en formación inicial

- Cuestionario de incidencias críticas

FIgURA 1. Adaptación del Ciclo de Aprendizaje de Karplus 
persigue alcanzar, su evolución y las prácticas educativas más favorables para su aprendizaje.

Para buscar respuesta a sus dudas y preguntas, se le pidió al alumnado que escribiese un artículo basado en publicaciones actuales sobre EAS, donde se estructurase la información recabada y contrastada. Para su fundamentación el alumnado debió realizar tareas de búsqueda y tratamiento de la información, análisis en base al currículo y consulta a personas expertas.

Después se llevó a cabo el ciclo para tratar la Sostenibilidad en el aula, que gravitó en torno a una simulación/role-play que conlleva una tarea de carácter investigativa de un problema socioambiental, por su potencial para explicitar valores y fomentar la participación democrática (Öhman \& Öhman, 2013; Todd, 2009), además de proporcionar una vivencia extraordinaria sobre la situación que se propone. Así, la propuesta planteó un debate sobre la instalación de una plataforma de Energía Eólica y su contribución a la mitigación del Cambio Climático. Aquí los equipos cooperativos, repartidos en los diferentes roles que se ven involucrados en la problemática, llevaron a cabo un análisis de la situación tratando cuestiones como: causas y consecuencias del Cambio Climático, posibles formas de abordar el problema (de carácter tecnocientífico/transformador social), ventajas y desventajas, consecuencias,..., y se inició una búsqueda de información en equipos, crítica y reflexiva, potenciando el análisis de la problemática desde diferentes perspectivas y su relación con las dimensiones socioculturales y económicas de nuestros estilos de vida.

El ciclo de aprendizaje culminó con una toma de decisiones consensuada y razonada, basada en dificultades y prioridades de acción. De esta forma, el alumnado concluyó que su comunidad podía contribuir a la producción energías alternativas, pero siendo consciente de las problemáticas que una solución tecnológica supone (generación dependiente de condiciones climáticas, producción de residuos,...). Esta conclusión conllevo, asimismo, el abordaje de la cuestión desde la responsabilidad individual y colectiva, analizándose aquellos aspectos cotidianos que podían ser modificados para facilitar el decrecimiento de la demanda energética.

Tras la realización de esta experiencia, se le pidió al alumnado que analizase globalmente $\mathrm{y}$ de forma fundamentada si esta experiencia educativa era adecuada para llevar a cabo en sus futuras aulas. En este momento se facilitó una reflexión sobre los diferentes modelos didácticos que podemos utilizar en el proceso de aprendizaje de la EAS, que buscaba contrastar las propias ideas con la propuesta realizada, donde se analizase críticamente la tendencia a reproducir los modelos de enseñanza experimentados (Garmendia Mujika et al., 2014). Con esta fase se perseguía facilitar la compresión y aplicación del marco educativo propio recién construido, revisando e integrando los nuevos conocimientos, de forma que se facilitase la evaluación del aprendizaje realizado y una nueva valoración conjunta en torno al proceso.

\subsection{IMPLEMENTACIÓN DELA PROPUESTA}

Esta intervención educativa se implementó a lo largo del segundo cuatrimestre de 2017 con un grupo de 25 maestras y maestros en formación, distribuidos en seis equipos cooperativos, que cursaban la materia opcional de "Educación Ambiental para el Desarrollo" en el Grado de Educación Primaria de la Facultad de Ciencias de la Educación y del Deporte de Pontevedra, de los cuales el $78 \%$ eran mujeres y el $22 \%$ eran hombres. En cuanto a la edad, un $66 \%$ tenían entre 18 y 22 años, un $24 \%$ entre 22 y 30 y un $10 \%$ más de 30 . 


\section{METOD OLOGÍA}

La investigación fue llevada a cabo con un enfoque socio-crítico (Robottom \& Hart, 1993), que persigue reflexionar y mejorar las prácticas cotidianas del futuro profesorado en relación con la EAS y los problemas socioambientales. En el estudio se utilizó un planteamiento metodológico mixto, ya que puede ayudar a confrontar la complejidad de este tipo de problemas de estudio y enriquecer su comprensión (Plano-Clark, Creswell, Green, \& Shope, 2008). De esta forma, datos cualitativos de distinta índole fueron utilizados para tratar de explicar y matizar los datos cuantitativos obtenidos con una escala tipo Likert (Irwin, 2008).

La escala utilizada, llamada Escala de Actitudes hacia la Educación Ambiental (ver anexo, Pérez-Rodríguez, Varela-Losada, Álvarez-Lires \& Vega-Marcote, 2017), presenta una buena consistencia interna $(\alpha=.804, \mathrm{glb}=.875 \mathrm{y}$ $\Omega=.810$ ) y proporciona información sobre las actitudes frente a un modelo educativo de la EAS de carácter transformador. La escala fue administrada de forma voluntaria antes y después de la intervención didáctica, siendo realizada por el $84 \%$ de las personas participantes. Los resultados de la escala fueron tratados con el programa SPSS versión 20 para Windows, utilizándose pruebas no paramétricas de significatividad.

Dos semanas después de desarrollar la experiencia didáctica, se llevaron a cabo entrevistas semiabiertas al futuro profesorado, donde se realizaron cuestiones relevantes con la práctica de una EAS y basadas en el marco utilizado en la construcción de la escala. Las entrevistas fueron realizadas a seis personas del grupo-clase, dos chicos y cuatro chicas, que llamaremos Boris, Pablo, Estefanía, Silvia, María e Inés, cuya selección se realizó según criterios de representatividad del grupo clase, teniendo en cuenta su género, su edad, su origen, su formación académica y su implicación en el aula. También se utilizó con todo el alumnado un cuestionario de incidencias críticas (Brookfield, 1995), donde se le pidió que valorase la experiencia, destacase el aprendizaje realizado y aportase propuestas de mejora. El análisis de estos datos fue combinado con los resultados obtenidos en la tarea final del ciclo de aprendizaje sobre EAS.

Los datos cualitativos fueron tratados mediante una metodología interpretativa (Erickson, 2012), usando análisis de contenido. Esta técnica es una delas más utilizadas para organizarla información obtenida y permite realizar inferencias, así como la identificación sistemática de las características específicas de los mensajes (Bardin, 1991). Con el objetivo de mejorar el análisis y comprensión de los resultados obtenidos, se agruparon los datos por temáticas similares para su triangulación. Estas categorías fueron seleccionadas a priori y su elección estuvo basada en una revisión de la literatura sobre problemáticas relacionadas con la formación del profesorado, que también se utilizó en la construcción de la escala (PérezRodríguez, Varela-Losada, Álvarez-Lires, \& Vega-Marcote, 2017).

\section{RESULTADOS Y DISCUSIÓN}

4.1 LA IMPORTANCIA DE LA INTEGRACIÓN DE LA EDUCACIÓN AMBIENTAL PARA LA SOSTENIBILIDAD EN LA EDUCACIÓN

Los resultados de la escala de actitudes mostraron el compromiso del alumnado con la integración de la EAS tanto en la escuela como en la formación del profesorado (ver Figura 2). Dato que también parece corroborar el cuestionario de incidencias críticas, donde el alumnado habla de la necesidad de cambio y de la importancia de formar al personal docente en este campo.

Asimismo en las entrevistas realizadas, las seis personas consultadas coincidieron en la importancia de integrar la EAS en la escuela, 


\section{Resultados de la escala de actitudes}

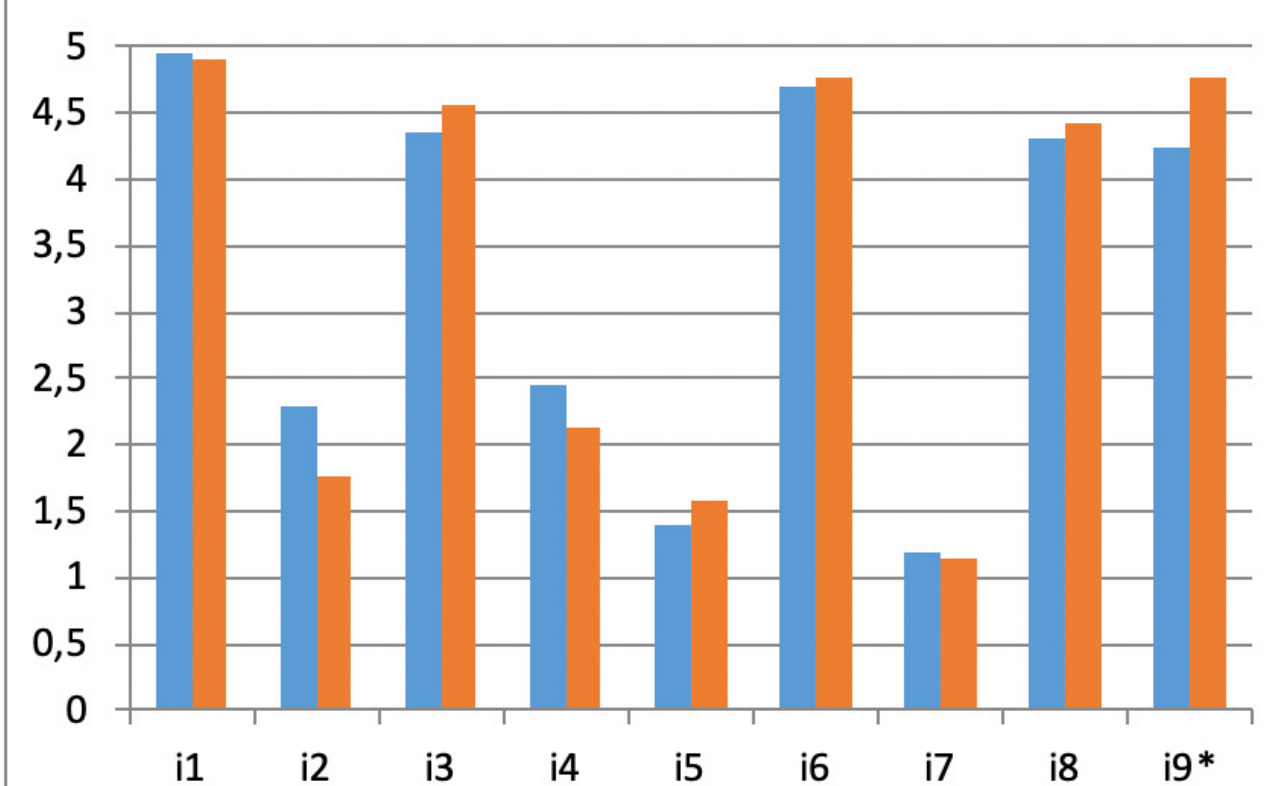

Antes de la intervención

Después de la intervención

FIGURA 2. Resultados obtenidos con la aplicación de la escala de actitudes antes y después de la intervención

Nota: Escala de actitudes con opciones del 1 al 5 según el grado de acuerdo o desacuerdo (ciertas cuestiones están presentadas de forma negativa), donde sólo un ítem mostró respuestas significativamente diferentes ${ }^{*} \mathrm{p}<.05$ (U de Mann-Whitney), debido probablemente a la baja capacidad de muestras pequeñas para detectar diferencias significativas.

"para aprender los efectos de nuestras acciones cotidianas" (Boris), "conocer qué podemos hacer" (Estefanía), o "por todo lo que hemos visto en clase, para conseguir ese compromiso de la ciudadanía con el planeta" (Inés). Aunque, cuando se les preguntó por su prioridad, una de las personas entrevistadas reconoció que hay ciertos contenidos (de lengua o matemáticas) que considera más importantes, coincidiendo con la tendencia del profesorado a ver la Educación Científica y la EAS como componentes poco prioritarios de los programas escolares (Marx \& Harris, 2006).

En este sentido, una excusa habitual para la falta de atención a la Educación Ambiental en la escuela suele ser la falta de tiempo para impartir todos los contenidos del extenso currículo (Evans, Whitehouse, \& Gooch, 2012). En nuestro caso, tres personas entre el futuro profesorado señalaron la importancia de cambiar la forma tradicional de organización de las materias, de forma que se pueda integrar el aprendizaje de contenidos de otras asignaturas con la EAS: "No creo que sea necesario quitar o poner horas, a lo mejor lo que se debería hacer es estructurarlo de forma diferente lo que se dedica a cada materia" (Boris). En el contexto de la conversación estas ideas parecen reflejar la necesidad de utilizar metodologías globalizadoras y holísticas.

\subsection{EL OBJETIVO PRINCIPAL DE LA EDUCACIÓN} AMBIENTAL ORIENTADA HACIA LA SOSTENIBILIDAD

En relación con su objetivo, la literatura muestra una preocupante discrepancia entre la filosofía de la EAS y la realidad de las prácticas escolares, que generalmente enfatizan la adquisición de conocimientos ambientales y la conciencia, 
cuando deberían centrarse en la resolución de problemas y en objetivos orientados hacia la acción (Mogensen \& Mayer, 2005; Stevenson, 2007).

En nuestro caso, trasllevar a cabolaintervención educativa, el análisis de la tarea realizada parecía mostrar que el alumnado asimiló correctamente los objetivos relacionados con la acción en la solución de los problemas socioambientales (todos los grupos defendieron este objetivo). Sin embargo los resultados de las entrevistas inducen a pensar que sigue persistiendo una tendencia a considerar la concienciación como la principal finalidad de la EAS. Así, Boris afirma que "tomar decisiones responsables viene de la mano de la concienciación" e Inés señala que "lo más importante es tener conocimientos sobre los problemas ambientales y concienciar", reflejando modelos que representan una simplificación excesiva de la realidad basados en la creencia de la relación causal más o menos lineal entre conciencia ambiental y comportamiento (Wals, Brody, Dillon \& Stevenson, 2014). Ideas más concordantes con los enfoques actuales mostraron María y Silvia, que defienden "educar para la acción,... crear actitudes y sentido crítico, capacidad de reflexionar sobre las cosas y no tomar todo como venga y ya está y aceptarlo", o "lo importante es ayudarles a pensar por sí mismos".

\subsection{LA PRÁCTICA EDUCATIVA EN LA EDUCACIÓN} AMBIENTAL ORIENTADA HACIALA SOSTENIBILIDAD

Un fin básico de la propuesta didáctica llevada a cabo era que el futuro profesorado vivenciase modelos alejados de la predominante práctica educativa transmisiva (Eames et al., 2006) y se cuestionase la reproducción de modelos de enseñanza tradicionales. El análisis de la propuesta realizado muestra que los equipos de futuros docentes defienden que la EAS debe ser reflexiva y crítica, globalizadora y problematizadora (ver figura 3), asociándola con características propias del enfoque que defiende la EAS actual. También relacionan su práctica con la resolución de problemas ambientales, el enfoque globalizador, la participación activa y el desarrollo de la autonomía (ver figura 3). Estos resultados coinciden con los datos que aportan tanto la escala de actitudes como las entrevistas.

De esta forma, tras la experiencia, todas las personas entrevistadas coincidieron en la adecuación de analizar y buscar soluciones para los problemas socioambientales en la etapa de Primaria, con apoyo del profesorado. Como Pablo resalta, "hay problemas que son fácilmente adaptables a cursos de Primaria, que sí se podrían trabajar o bien en el aula o bien en el entorno".

Además se les preguntó específicamente si no sería más provechoso facilitarles los contenidos ya seleccionados y analizados, con el fin de analizar si defendían un aprendizaje autónomo basado en la búsqueda de la información y su reflexión crítica. Todos y todas coincidieron en la importancia de fomentar este tipo aprendizaje donde el alumnado tratase por sí mismo la información. Como Boris e Inés reconocen, "Hoy en día es muy importante que aprendan a buscar y a diferenciar entre qué información es válida y cual no", "que busquen información yo creo que les vale para todo además, para toda su formación", reconociendo su importancia para la alfabetización científica y ciudadana (Oliveras, Márquez, \& Sanmartí, 2013).

Desde la perspectiva globalizadora, la investigación delos problemassocioambientales en el aula implica reflexionar sobre las influencias culturales, socioeconómicas y políticas en nuestros comportamientos (Gifford, 2014; Pérez-Rodríguez, VarelaLosada, Lorenzo-Rial, \& Vega-Marcote), y por tanto tratar el modelo socioeconómico 


\section{Características de la Educación Ambiental}
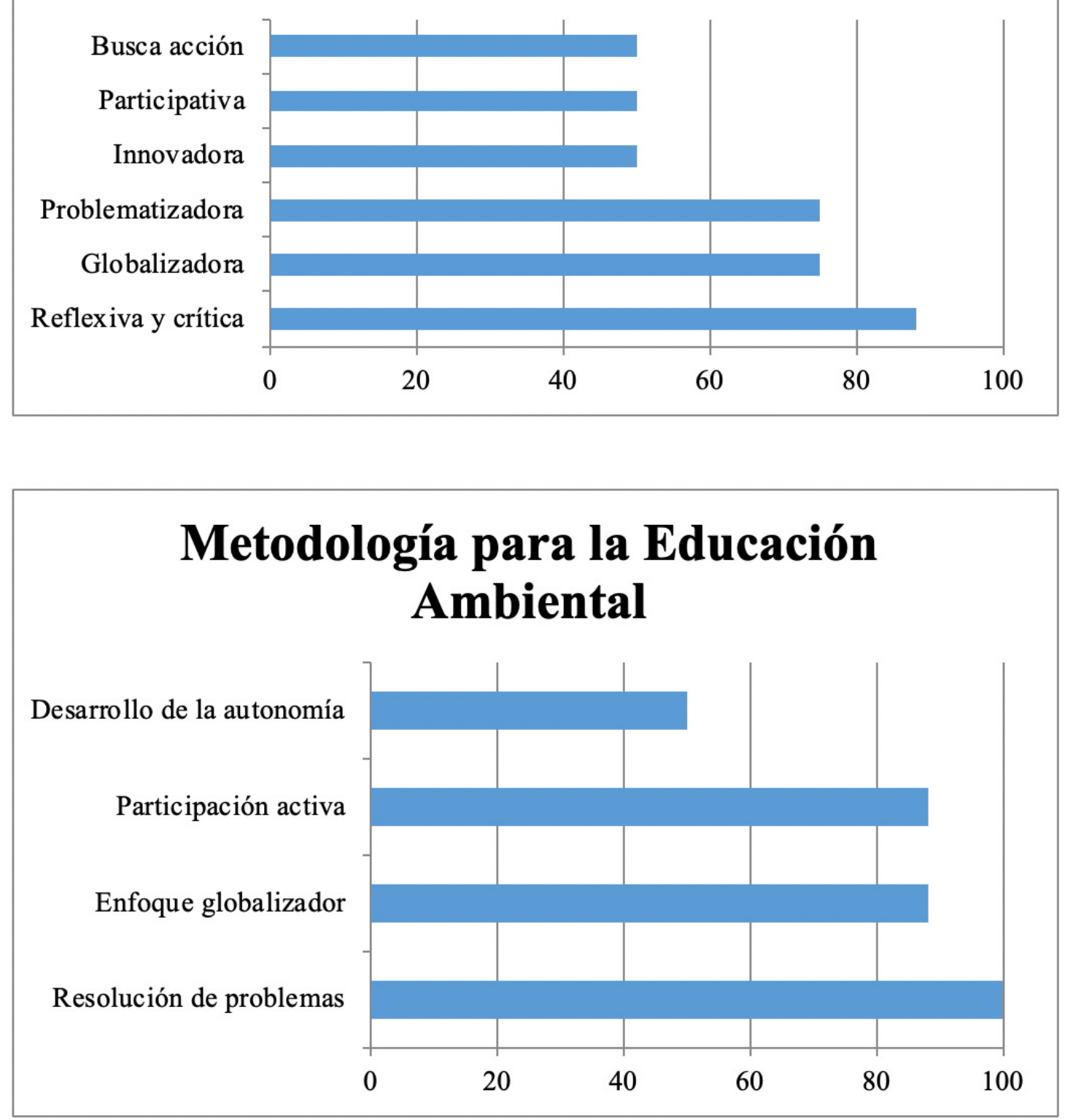

FIGURA 2. Resultados que defiende el alumnado, tras la experiencia didáctica, para la práctica de EAS.

actual basado en el consumo (Álvarez-Suárez, Vega-Marcote, \& Garcia-Mira, 2014). Así, cinco de las personas entrevistadas estuvieron de acuerdo en la importancia de introducir esta temática en el aula de forma adaptada. Inés dice: "es que se supone que ese el origen, ¿no? Es uno de los factores más importantes. Sí que se debe trabajar en el aula", resultados que también parecen corroborar los datos obtenidos a través de la escala. 


\subsection{La IMPLICACIÓN DE LA COMUNIDAD}

En relación con la implicación del profesorado y de la comunidad, los datos aportados por la escala (ver Figura 1) y por las entrevistas mostraron resultados prometedores. Así, todas las personas entrevistadas estuvieron de acuerdo en la importancia del profesorado como modelo para su alumnado. Lo mismo ocurre con la implicación de la comunidad, que las seis personas entrevistadas valoraron muy positivamente aunque señalando distintas razones. Inés y María destacaron la influencia de las familias en su alumnado: "si eres niño, cuanto más compromiso veas, si ves que hay más agentes, no solo tú y tus compañeros, pues eso es como más importante". Y Pablo y Boris lo fundamentaron basándose en que es un factor fundamental para la solución de los problemas colectivos: "más que nada, para llevar a cabo proyectos a gran escala ósea; si lo hacemos sólo el profesor y los alumnos, queda como muy... limitado a nuestro entorno, pero si lo ampliamos a familias, si lo ampliamos a todo el colegio, siempre vamos a poder tener más posibilidades de llevar a cabo proyectos a gran escala que a la larga van a ayudar más".

A pesar de percibir esta importancia, tres chicas señalaron la dificultad/imposibilidad de conseguir este compromiso del entorno. Así, María dice: "yo creo que eso es complicado, quizá porque siempre que algo depende más allá de ti, que eres la profesora en el aula, todo se complica más, sobre todo con las familias, pero sería lo ideal, porque aparte si eres niño, cuanto más compromiso veas, si ves que hay más agentes, no solo tú y tus compañeros, pues eso es como más importante”. De esta forma, este futuro profesorado intuye las limitaciones organizativas y sociales a las que suele tener que enfrentarse el personal docente que se esfuerza por adoptar una postura social crítica (Walker, 1997).
Cuando se les pregunta si la escuela puede contribuir a cambiar el comportamiento de la comunidad encontramos disparidad de opiniones. Cuatro de las personas entrevistadas defienden que sí pero en un futuro, cuando su alumnado se convierta en personas adultas, reflejando una de las dificultades con las que tropieza la implicación de la ciudadanía en el avance hacia la Sostenibilidad, que es la creencia errónea de que dicha transición es un objetivo para el futuro (Vilches \& Gil Pérez, 2016). Sin embargo, María y Silvia confían en la influencia "que los niños tienen ahora en sus familias" y en su poder como agentes de cambio, cuyo efecto ha sido descrito por autores como Schneller (2008). Quizás el aprendizaje más importante es que todo el alumnado entrevistado parece ser consciente de que el proceso de enseñanza de la EAS es mejor cuando implicamos a las comunidades en él, tal como muestran algunos estudios (Schelly, Cross, Franzen, Hall, \& Reeve, 2012).

\subsection{EVALUACión DEL PROCESO}

Los datos obtenidos mediante el cuestionario de incidencias críticas, señalado anteriormente, muestran que el alumnado valoró muy positivamente la experiencia llevada a cabo, destacando el cambio de visión aportado y la forma de plantear la experiencia, fomentando la reflexión y el pensamiento crítico, y facilitando el compromiso con la EAS. Además muchos de sus comentarios reflejaron lo aprendido sobre la importancia de trabajar metodologías que permitan pensar críticamente, reflexionar sobre nuestras acciones y participar.

El seguimiento del proceso por el profesorado sugirió, de igual forma, que la experiencia fue llevada a cabo de forma positiva y reflejó como el alumnado mostró motivación e interés en colaborar en todas la tareas realizadas, y tras la experiencia se percibió una evolución 
en la capacidad de analizar críticamente la información, de reflexión y de implicación en el debate socioambiental.

No obstante, también es necesario reflexionar sobre algunos de los resultados obtenidos. La investigación induce a pensar que una parte del futuro profesorado sigue percibiendo la concienciación como el objetivo principal de la EAS, lo que puede ser un primer paso hacia la reflexión crítica de nuestra forma de vida, pero que no siempre implica el fomento de la acción participativa en la búsqueda de soluciones de los problemas socioambientales. Además los datos también parecen evidenciar resultados perfectibles sobre la importancia de la implicación de la comunidad, que puede mejorar su percepción sobre la importancia de pensar en la acción participativa como la finalidad principal de la EAS, y la necesidad de contribuir a una reconceptualización del sistema educativo actual, integrando objetivos de conservación, justicia social, desarrollo sostenible y democracia (Tilbury, Coleman, \& Garlick, 2005).

\section{CONCLUSIONES}

Actualmente es vital impulsar la transición hacia escuelas y comunidades comprometidas con la conservación del medio y el bienestar de las personas. En este proceso el profesorado es un factor clave y su formación tiene un efecto multiplicador a través de su futura carrera profesional.

La propuesta evaluada aborda la cuestión partiendo de la importancia de vivenciar experiencias educativas innovadoras que permitan al alumnado reflexionar sobre nuevos enfoques en el proceso de enseñanza yaprendizaje. Los resultados muestran que este tipo de prácticas basadas en el aprendizaje experiencial y la reflexión didáctica pueden contribuir a mejorar el compromiso del futuro profesorado con una
EAS y crítica con el modelo actual de desarrollo, poniendo los cimientos para el desarrollo de competencias docentes fundamentales, como la comprensión de la necesidad de transformar los sistemas educativos y la manera en que educamos/aprendemos, la capacidad de implicar al alumnado en cuestiones reales y tratar problemas con distintas perspectivas, o la capacidad de fomentar el intercambio de ideas y la clarificación de sus propias visiones del mundo (United Nations Economic Commission for Europe [UNECE], 2013). De esta forma, facilitan la construcción de un marco educativo basado en metodologías globalizadas y participativas, que busca la formación de personas autónomas y reflexivas, capaces de utilizar la información de forma crítica para colaborar en la resolución de problemas socioambientales e implicando a la comunidad.

También, cabe destacar que se percibe una gran evolución del aprendizaje, ya que inicialmente el alumnado no identificaba explícitamente el modelo socioecónomico basado en el consumo como un contenido clave de la EAS, y que puede ser fundamental para fomentar el cambio en los estilos de vida de las personas. Aunque, también es necesario señalar determinadas limitaciones, como la evolución positiva en las actitudes del futuro profesorado, debido a que, en muchos casos, los niveles actitudinales eran de partida muy altos (tal como también reflejan los barómetros europeos sobre el mismo tema), y había poco margen de mejora, o la duración del estudio. Además debemos tener en cuenta que se trata de una experiencia con un número reducido de participantes, por lo que sería muy pertinente ampliar la muestra en futuras investigaciones. También sería relevante continuar estudiando en profundidad la capacidad del alumnado para argumentar una toma de decisiones fundamentada.

En conclusión, es necesario continuar con esta línea de investigación en futuros trabajos que 
busquen mejorar la formación del profesorado en torno a la EAS, de forma que esta pueda ser la base de nuevas propuestas que persigan educar una ciudadanía que sea capaz de afrontar los retos socioambientales presentes y futuros.

\section{REFERÊ NCI A S}

Agirreazkuenaga, L. (2019). Embedding sustainable development goals in education. Teachers' perspective about education for sustainability in the Basque Autonomous Community. Sustainability, 11(5), 1496. doi: 10.3390/su11051496

Alarcão, I., Tavares, J., Mealha, O., \& Souza, D. (2018). Pensar a universidade dos próximos 20 anos através de uma metodologia de cenários. Revista Portuguesa de Educação, 31(1), 108122. doi: $10.21814 /$ rpe. 12622

Álvarez-Suárez, P., Vega-Marcote, P., \& GarciaMira, R. (2014). Sustainable consumption: A teaching intervention in higher education. International Journal of Sustainability in Higher Education, 15(1), 3-15. doi: 10.1108/ IJSHE-06-2011-0044

Arreguín-Anderson, M., \& Kennedy, K. (2013). Deliberatelanguage planning in environmental education: A CRT/LatCrit perspective. The Journal of Environmental Education, 44 (1), 1-15. doi: 10.1080/00958964.2012.665098

Bardin, L. (1991). Análisis de contenido. Madrid, España: Ediciones Akal.

Borg, N., Gericke, H., Höglund, O., \& Bergman, E. (2014). Subject and experience bound differences in teachers' conceptual understanding of sustainable development. Environmental Education Research, 20(4), 526551.

Brookfield, S. (1995). Becoming a critically reflective teacher. San Francisco: Jossey-Bass.

Cebrián, G., \& Junyent, M. (2015). Competencies in education for sustainable development: Exploring the student teachers' views. Sustainability, 7(3), 2768-2786.

Desjean-Perrotta, B., Moseley, C., \& Cantu, L.
E. (2008). Preservice teachers' perceptions of the environment: Does ethnicity or dominant residential experience matter?. The Journal of Environmental Education, 39(2), 21-32.

Eames, C., Law, B., Barker, M., Iles, H., McKenzie, J., Patterson, R., ... \& Wright, A. (2006). Investigating teachers' pedagogical approaches in environmental education that promote students' action competence. Wellington, New Zealand: Teaching \& Learning Research Initiative. Retrieved from http://www.tlri. org.nz/sites/default/files/projects/9224 finalreport.pdf

Erickson, F. (2012). Qualitative research methods for science education. In B. J. Fraser, K. Tobin, \& C. J. McRobbie (Eds.), Second international handbook of science education (pp. 1451-1469). Netherlands: Springer.

Eurydice. (2011). La enseñanza de las Ciencias en Europa: Políticas nacionales, prácticas $e$ investigación. Centro de Publicaciones. Ministerio de Educación, Cultura y Deporte. Centro Nacional de Innovación e Investigación Educativa (CNIIE). Madrid: Secretaría General Técnica.

Evans, N., Whitehouse, H., \& Gooch, M. (2012). Barriers, successes and enabling practices of education for sustainability in far North Queensland schools: A case study. The Journal of Environmental Education, 43(2), 121-138.

Feinstein, N. W., \& Kirchgasler, K. L. (2015). Sustainability in science education? How the Next Generation Science Standards approach sustainability, and why it matters. Science Education, 99(1), 121-144. doi: 10.1002/ sce. 21137

Fernández, M., Alférez, A., Vidal, S., Fernández, M. Y., \& Albareda, S. (2016). Methodological approaches to change consumption habits of future teachers in Barcelona, Spain: Reducing their personal ecological footprint. Journal of Cleaner Production, 122, 154-163. doi: 10.1016/j.jclepro.2016.02.026

Fortner, R. W., \& Corney, J. R. (2002). Great Lakes educational needs assessment: Teachers' 
priorities for topics, materials, and training. Journal of Great Lakes Research, 28(1), 3-14.

Foss, A. W., \& Ko, Y. (2019). Barriers and opportunities for climate change education: The case of Dallas-Fort Worth in Texas. The Journal of Environmental Education, 50(3), 145-159. doi: 10.1080/00958964.2019.1604479

García-Esteban, F. E., \& Murga-Menoyo, A. (2015). El profesorado de educación infantil ante el desarrollo sostenible. Necesidades formativas. Enseñanza \& Teaching: Revista interuniversitaria de didáctica, 33(1), 121-142. doi: 10.14201/et2014331121142

Garmendia Mujika, M., Barragués Fuentes, J. I., Zuza Elosegi, K., \& Guisasola Aranzabal, J. (2014). Proyecto de formación del profesorado universitario de Ciencias, Matemáticas y Tecnología, en las metodologías de Aprendizaje Basado en Problemas y Proyectos. Enseñanza de las Ciencias, 32(2), 113-129. doi: 10.5565/ rev/ensciencias.911

Gayford, C. (2001). Education for sustainability: An approach to the professional development of teachers. European Journal of Teacher Education, 24(3), 313-327.

Gifford, R. (2014). Environmental psychology matters. Psychology, 65(1), 541-579. doi: 10.1146/annurev-psych-010213-115048

Irwin, S. (2008). Data analysis and interpretation. In C. Hesse-Biber \& P. Leavy (Eds.), Handbook of emergent methods (pp. 415-436). New York: Guilford Press.

Karplus, R. (1977). Science teaching and the development of reasoning. Journal of Research in Science Teaching, 14(2), 169-175.

Kopnina, H. (2012). Education for sustainable development (ESD): The turn away from "environment" in environmental education? Environmental Education Research, 18(5), 669717.

Kyburz-Graber, R. (2013) Socioecological approaches to environmental education and research: A paradigmatic response to behavioral change orientations. In R. B. Stevenson, M. Brody, J. Dillon, \& A. E. J. Wals
(Eds.), International handbook of research on environmental education (pp. 23-32). New York: Routledge.

Lave, J., \& Wenger, E. (1991). Situated learning: Legitimate peripheral participation. Cambridge, United Kingdom: Cambridge University Press.

Lotz-Sisitka, H., Wals, A. E., Kronlid, D., \& McGarry, D. (2015). Transformative, transgressive social learning: Rethinking higher education pedagogy in times of systemic global dysfunction. Current Opinion in Environmental Sustainability, 16, 73-80. doi: 10.1016/j.cosust.2015.07.018

Marx, R. W., \& Harris, C. J. (2006). No Child Left Behind and science education: Opportunities, challenges, and risks. The Elementary School Journal, 106(5), 467-478.

Mascarell, L., \& Vilches Peña, A. (2016). Química verde y sostenibilidad en la educación en ciencias en secundaria. Enseñanza de las Ciencias, 34(2), 25-42. doi: 10.5565/rev/ ensciencias. 1688

McKeown, R., \& Hopkins, C. (2002). Weaving sustainability into pre-service teacher education programs. In W. Leal Filho (Ed.), Teaching sustainability at universities: Towards curriculum greening (pp. 251-274). Frankfurt, Germany: PeterLang.

Mogensen, F., \& Mayer, M. (2005). Eco-schools: Trends and divergences. A comparative study on ECO-school development processes in 13 countries. Viena, Austria: Austrian Federal Ministry of Education, Science and Culture.

Naciones Unidas. (2015). Transformar nuestro mundo: La agenda 2030 para el desarrollo sostenible. Disponible en http://www.un.org/ es/comun/docs/?symbol=A/RES/70/1

O 'Gorman, L., \& Davis, J. (2013). Ecological footprinting: Its potential as a tool for change in preservice teacher education. Environmental Education Research, 19(6), 779-791.

Öhman, J., \& Öhman, M. (2013). Participatory approach in practice: An analysis of student discussions about climate change. 
Environmental Education Research, 19(3), 324341.

Oliveras, B., Márquez, C., \& Sanmartí, N. (2013). The use of newspaper articles as a tool to develop critical thinking in science classes. International Journal of Science Education, 35(6), 885-905.

Pérez-Rodríguez, U., Varela-Losada, M., ÁlvarezLires, F. J., \& Vega-Marcote, P. (2017). Attitudes of preservice teachers: Design and validation of an attitude scale toward environmental education. Journal of Cleaner Production, 164, 634-641. doi: 10.1016/j.jclepro.2017.06.245

Pérez-Rodríguez, U., Varela-Losada, M., Lorenzo-Rial, M. A., \& Vega-Marcote, P. (2017). Tendencias actitudinales del profesorado en formación hacia una educación ambiental transformadora. Revista de Psicodidáctica, 22(1), 60-68. doi: 10.1016/ S1136-1034(17)30045-X

Piasentin, F. B., \& Roberts, L. (2018). What elements in a sustainability course contribute to paradigm change and action competence? A study at Lincoln University, New Zealand. Environmental Education Research, 24(5), 694715. doi: 10.1080/13504622.2017.1321735

Plano-Clark, V. L., Creswell, J. W., Green, D. O. N., \& Shope, R. J. (2008). Mixing quantitative and qualitative approaches. In C. Hesse-Biber \& P. Leavy (Eds.), Handbook of emergent methods (pp. 363-388). New York: Guilford Press.

Robottom, I., \& Hart, P. (1993). Research in environmental education: Engaging the debate. Geelong, Victoria, Australia: Deakin University.

Schelly, C., Cross, J., Franzen, W., Hall, P., \& Reeve, S. (2012). How to go green: Creating a conservation culture in a public high school through education, modelling, and communication. The Journal of Environmental Education, 43(3), 143-161. doi: 10.1080/00958964.2011.631611

Schneller, A. (2008). Environmental service learning: Outcomes of innovative pedagogy in Baja California Sur, Mexico. Environmental Education Research, 14(3), 291-307.

Steffen, W., \& Stafford, M. (2013). Planetary boundaries, equity and global sustainability: Why wealthy countries could benefit from more equity. Current Opinion in Environmental Sustainability, 5, 403-408. doi: 10.1016/j. cosust.2013.04.007

Stern, P. C. (2000). Toward a coherent theory of environmentally significant behavior. Journal of Social Issues, 56, 407-424.

Stern, M. J., Powell, R. B., \& Hill, D. (2014). Environmental education program evaluation in the new millennium: What do we measure and what have we learned?. Environmental Education Research, 20(5), 581-611. doi: 10.1080/13504622.2013.838749

Stevenson, R. B. (Ed.). (2007). Revisiting schooling and environmental education: Contradictions in purpose and practice [Editorial]. Environmental Education Research, 13(2), 129-138.

Sund, P., \& Wickman, P. O. (2011). Socialization content in schools and education for sustainable development: A study of teachers' selective traditions. Environmental Education Research, 17(5), 599-624.

Tilbury, D. (2011). Education for sustainable development. An expert review of processes and learning. Paris, France: UNESCO. Retrieved from http://unesdoc.unesco.org/ images/0019/001914/191442e.pdf

Tilbury, D., Coleman, V., \& Garlick, D. (2005). A National review of environmental education and its contribution to sustainability in Australia: school education. (A National Review of Environmental Education and its Contribution to Sustainability in Australia). Canberra, ACT: Department of the Environment and Heritage. Todd, S. (2009). Toward an imperfect education: Facing humanity, rethinking cosmopolitanism. Boulder, Colorado: Paradigm.

United Nations Economic Commission for Europe. (2013). Empowering educators for a sustainable future. Geneva, Switzerland: 
United Nations Economic Commission for Europe. Retrieved from https://www. unece.org/fileadmin/DAM/env/esd/ESD Publications/Empowering Educators for a Sustainable Future ENG.pdf

Van Petegem, P., Blieck, A., \& Van Ongevalle, J. (2007) Conceptions and awareness concerning environmental education: A Zimbabwean case-study in three secondary teacher education colleges. Environmental Education Research, 13(3), 287-306.

Varela-Losada, M., Arias-Correa, A., \& VegaMarcote, P. (2018). Training teachers committed to climate change mitigation. In U. M. Azeiteiro, W. Leal Filho, \& L. Aires (Eds.), Climate literacy and innovations in climate change education (pp. 307-321). Cham, Switzerland: Springer.

Varela-Losada, M., Vega-Marcote, P., Pérez-Rodríguez, U., \& Álvarez-Lires, M. (2016). Going to action? A literature review on educational proposals in formal environmental education. Environmental Education Research, 22(3), 390-421. doi: 10.1080/13504622.2015.1101751

Vilches, A., \& Gil Pérez, D. (2016). La transición a la sostenibilidad como objetivo urgente para la superación de la crisis sistémica actual. Revista Eureka sobre Enseñanza y Divulgación de las Ciencias, 13(2), 395-407. Disponible en http://hdl.handle.net/10498/18296

Vosniadou, S. (2001). How children learn. Brussels, Belgium: International Academy of Education.

Walker, K. (1997). Challenging critical theory in environmental education. Environmental Education Research, 3(2), 155-162.

Wals, A. (Ed.). (2007). Social learning towards a sustainable world. Wageningen, Netherlands: Wageningen Academic.

Wals, A. E., Brody, M., Dillon, J., \& Stevenson R. B. (2014). Convergence between science and environmental education. Science, 344(6184), 583-584. doi: 10.1126/science.1250515

Worldwatch Institute. (2015). Confronting hidden threats to sustainability: State of the world 2015. Washington: The Worldwatch Institute.

Yavetz, B., Goldman, D., \& Pe’er, S. (2009). Environmental literacy of preservice teachers in Israel: A comparison between students at the onset and end of their studies. Environmental Education Research, 15(4), 393-415. 


\section{EDUCATE FOR CHANGE AND SUSTAINABILITY: EVALUATION OF AN EXPERIENTIALLEARNING PROPOSAL TO TRA IN TEACHERS IN IN IT IAL T R A IN IN G}

\section{Abstract}

One of the main challenges that education must face is the search for Sustainability. And, in this process, the teaching staff is a fundamental pillar. Therefore, an educational proposal of initial teacher training based on experimentation and analysis of a learning cycle around the climate change socioenvironmental problem is presented. For its evaluation, a mixed methodology was used. The results suggest that this type of practice can contribute to improve the commitment of the future teachers with an Environmental Education oriented towards Sustainability and critical with the current development model, allowing the construction of an educational framework based on globalized and participatory methodologies, as well as to favor the acquisition of teaching competences.

KEYwORDS: Environmental Education and Sustainability; Teacher training; Experiential Learning; Learning cycle
EDUCAR PARA A MUDANÇA E A SUSTENTABILIDADE: AVALIAÇÃO DE UMA PROPOSTA DE APRENDIZAGEM EXPERIENCIAL PARA CAPACITAR OS PROFESSORES EM FORMAÇÃO IN ICIAL

\section{RESUMEN}

Um dos principais desafios que a educação deve enfrentar é a busca pela Sustentabilidade. $\mathrm{E}$, nesse processo, o corpo docente é um pilar fundamental. Para tanto, apresenta-se uma proposta educacional de formação inicial de professores baseada na experimentação e análise de um ciclo de aprendizagem em torno do problema socioambiental da mudança climática. Para a sua avaliação, foi utilizada uma metodologia mista. Os resultados obtidos sugerem que esse tipo de prática pode contribuir para melhorar o comprometimento dos futuros professores com uma Educação Ambiental voltada à Sustentabilidade e crítica ao atual modelo de desenvolvimento, permitindo a construção de um arcabouço educacional baseado em metodologias globalizadas e participativas, bem como para favorecer a aquisição de competências de ensino.

Palavras-Chave: Educação Ambiental e Sustentabilidade; Formação de professores; Aprendizagem experiencial; Ciclo de aprendizagem

\footnotetext{
${ }^{I}$ Facultade de Ciencias da Educación e do Deporte. Universidade de Vigo, España. ORCID: 0000-0002-2752-4723

${ }^{\text {II }}$ Facultade de Ciencias da Educación e do Deporte. Universidade de Vigo, España. ORCID: 0000-0003-2508-4370 ${ }^{\text {III }}$ Facultade Ciencias da Educación. Universidade da Coruña, España. ORCID: 0000-0002-5776-7419
} 
ANEXO 1

Escala de Actitudes hacia la Educación Ambiental (de ahora en adelante EA)

(Pérez-Rodríguez, Varela-Losada, Álvarez-Lires \& Vega-Marcote, 2017)

i1 Ante los problemas ambientales de nuestro tiempo es prioritario integrar la EA en la escuela.

i2* Considero que no es prioritario que la EA trate el modelo socioeconómico actual basado en el consumo.

i3 La EA debería trabajar especialmente el desarrollo de habilidades como el pensamiento crítico, la toma de decisiones reflexivas y la participación.

i4* Considero que analizar problemas ambientales y buscar soluciones es demasiado complejo para alumnado de Primaria.

i5* El alumnado pierde demasiado tiempo en buscar y analizar la información, es mucho más provechoso facilitarles la información ya seleccionada y analizada.

i6 Para que la EA sea lo más efectiva posible debería existir un compromiso de toda la comunidad educativa.

i7* No me parece que el comportamiento del profesorado sea un factor muy importante en el aprendizaje de valores ambientales.

i8 Creo que incluir la EA en la escuela puede contribuir a cambiar el comportamiento ambiental de toda la comunidad.

i9 Me parece importante que todo el profesorado reciba formación ambiental.

*Los asteriscos indican que el ítem se ha formulado en sentido negativo. 